\title{
Energy dependence of strangeness production
}

\author{
Volker Friese \\ Gesellschaft für Schwerionenforschung, Planckstr. 1, 64291 Darmstadt, Germany \\ E-mail: v.friese@gsi.de
}

for the NA49 Collaboration*

\begin{abstract}
.
We present a summary of strange particle yields measured by the experiment NA49 in central $\mathrm{Pb}+\mathrm{Pb}$ collisions in the beam energy range $20-158 \mathrm{AGeV}$. The comparison with thermal hadron model results shows that above $30 \mathrm{AGeV}$, a strangeness undersaturation parameter $\gamma_{S}$ must be employed to describe the data. This suggests a change in the strangeness production mechanism at this energy. We find that the UrQMD transport model in all cases underpredicts the observed yield ratios.
\end{abstract}

Submitted to: J. Phys. G: Nucl. Phys.

\section{Introduction}

The experimental data accumulated by several experiments for $\mathrm{Pb}+\mathrm{Pb}$ collisions at top SPS energy $(158 \mathrm{AGeV})$ suggest that in the early stage of central reactions of this type a deconfined state of matter is created $[1,2]$. It is commonly believed that at lower beam energies, e.g. at SIS and AGS, such a phase transition does not take place. Both statements, although lacking a rigorous proof, motivate a search for the onset of deconfinement at energies between top AGS (11.7 AGeV) and top SPS energy. The NA49 collaboration has undertaken such a search by investigating central $\mathrm{Pb}+\mathrm{Pb}$ collisions at beam energies between $20 \mathrm{AGeV}$ and $158 \mathrm{AGeV}$.

One of the observables possibly signalling a transient deconfined state in the course of the collision is the relative yield of strange particles. Originally, it was proposed that strangeness is more easily produced in a Quark-Gluon-Plasma than in a purely hadronic system, leading to an enhancement of relative strangeness yields in heavy ion collisions in comparison to elementary $(p+p)$ reactions once the system underwent a phase transition [3]. The interpretation of the observed strange particle yields, however, turned out to be more complicated. Statistical hadron gas models were successful in * for the collaboration list see [11] 
describing the measured yields, suggesting an equilibrated hadronic system at chemical freeze out [4]. In such models, the strangeness enhancement in $\mathrm{Pb}+\mathrm{Pb}$ collisions is a consequence of the increased system volume, relaxing the constraints imposed by flavour conservation which result in a suppression of strangeness in small systems $[5,6]$. It is still under debate whether an equilibrium description also holds for strange particles, i.e. whether a strangeness undersaturation factor $\gamma_{S}$ is needed to describe the data [7]. This factor could be interpreted as the influence of a prehadronic stage onto the available hadronic phase space [8]. More data at various beam energies and collision system sizes will help to answer such questions.

As an early result of the NA49 energy scan programme, a sharp maximum in the $K^{+} / \pi^{+}$excitation function at $30 \mathrm{AGeV}\left(\sqrt{s_{N N}}=7.6 \mathrm{GeV}\right)$ was reported in the last conference of this series [9], consistent with the assumption of the onset of deconfinement at that energy [10]. Meanwhile, the energy scan has been completed by taking data at the lowest SPS energy $(20 \mathrm{AGeV})$. In addition, the available data have been analysed with respect to the production of other strangeness carriers. We present a summary of results obtained in the context of the energy scan programme by NA49 so far and compare them to data obtained at AGS and RHIC and to predictions of statistical and microscopic models. We concentrate on particle yields; a discussion of the kinematic distributions can be found in [11]. We choose UrQMD [12,13] as a representative of hadronic transport models. As thermal models we employ versions with and without the assumption of strangeness saturation. The former, in the following labelled as Hadron Gas Model (HGM), has predictive power by parametrising the variation of $T$ and $\mu_{B}$ with collision energy [14]. The latter, denoted by Statistical Hadronisation Model (SHM), fits the observed yields using $\gamma_{S}$ to describe the deviation of strange particle yields from full equilibrium [15].

In the context of hadron gas models, it is debated whether particle yields around midrapidity or fully integrated over rapidity should be considered. In the former case, one stays away from the fragmentation regions where the properties of the system may be different from those in the central region. However, the data obtained at AGS, SPS and even RHIC reveal nearly Gaussian rapidity distributions for the produced particles [16], showing no sign of a clear separation of central and fragmentation regions. Moreover, by using phase space integrated yields, the fit results are independent of the kinematical behaviour of the system as e.g. flow. By studying integrated yields, as we choose to do in this report, one of course addresses the average properties of the system (the "Equivalent Global Cluster" [15]) and is not sensitive to possible variations of thermal parameters with rapidity.

\section{Experiment and data analysis}

The NA49 experiment [17] is a large acceptance hadron spectrometer operated at CERN-SPS with external heavy ion beams. It consists of two superconducting dipole magnets and four large-volume TPCs for tracking and momentum measurement. The 
identification of charged particles is achieved by the measurement of specific energy loss in the TPC gas and, in a restricted acceptance region around midrapidity, by timeof-flight measurement in two scintillator arrays located at $14 \mathrm{~m}$ from the target. The event centrality is determined from the energy deposited by the beam spectators in a zero-degree calorimeter. For the data sets presented here, the online centrality trigger selected the $7 \%$ most central events except at $158 \mathrm{AGeV}$, where the centrality was $5 \%$ for kaons and $\phi, 10 \%$ for $\Lambda$ and $\Xi$ and $23.5 \%$ for $\Omega$. The data at lower energies $(20,30$, 40 and $80 \mathrm{AGeV}$, corresponding to $\sqrt{s_{N N}}=6.3,7.6,8.8$ and $12.3 \mathrm{GeV}$, respectively) were taken in the years 1999, 2000 and 2002, the data at top SPS energy $\left(\sqrt{s_{N N}}=17.3\right.$ $\mathrm{GeV}$ ) in 1996 and 2000.

For the identification of charged particles, TOF is used at momenta below $2.5 \mathrm{GeV}$ to separate pions, kaons and protons. Between 2.5 and $10 \mathrm{GeV}$, but still at midrapidity, the combined TOF and $d E / d x$ measurements still allow a clean separation almost on a track-by-track basis. At higher momenta, outside the TOF acceptance, the contributions of the particle species to the energy loss spectrum are resolved on a statistical basis [9].

Hyperons are identified in the invariant-mass spectrum of their decay daughters, using the decay topology to suppress the combinatorial background. Resonance signals are also extracted from the invariant-mass spectrum, which is constructed after appropriate PID cuts on their decay products.

The strange particle yields presented in the next sections will be normalised to the total pion yield $\langle\pi\rangle=1.5 \times\left(\left\langle\pi^{+}\right\rangle+\left\langle\pi^{-}\right\rangle\right)$. The $\pi^{-}$yield was derived by subtracting the contributions of $K^{-}$, secondary interactions and feeddown from weak decays from

the negatively charged hadron yield measured by NA49. From this, the $\pi^{+}$yield was calculated using the $\pi^{+} / \pi^{-}$ratio that was determined in the limited TOF acceptance. The method is described in detail in [18]. It should be noted that the pion yields at AGS and SPS are corrected for the contributions of weak decays. This does not hold for the RHIC data, where this contribution is estimated to be $4 \%$ [16].

\section{Results}

\subsection{Kaons}

The transverse mass spectra for $K^{+}$and $K^{-}$, measured at midrapidity in the TOF acceptance for the five beam energies show, within the experimental uncertainties, no deviations from an exponential shape. The slope parameters obtained by an exponential fit are similar for both particle types and do not vary significantly with beam energy [19]. The acceptance covers more than $95 \%$ of the total yield. The rapidity distributions, obtained by integration of the transverse spectra in different rapidity bins, can be described by the sum of two Gaussians displaced symmetrically around midrapidity [11]. For the $K^{-}$, a single Gaussian fit is equally satisfying. At larger beam energies, the TOF points at midrapidity agree well with the results obtained by the $d E / d x$ analysis, showing the consistency of these two identification methods. At the lower energies, there 

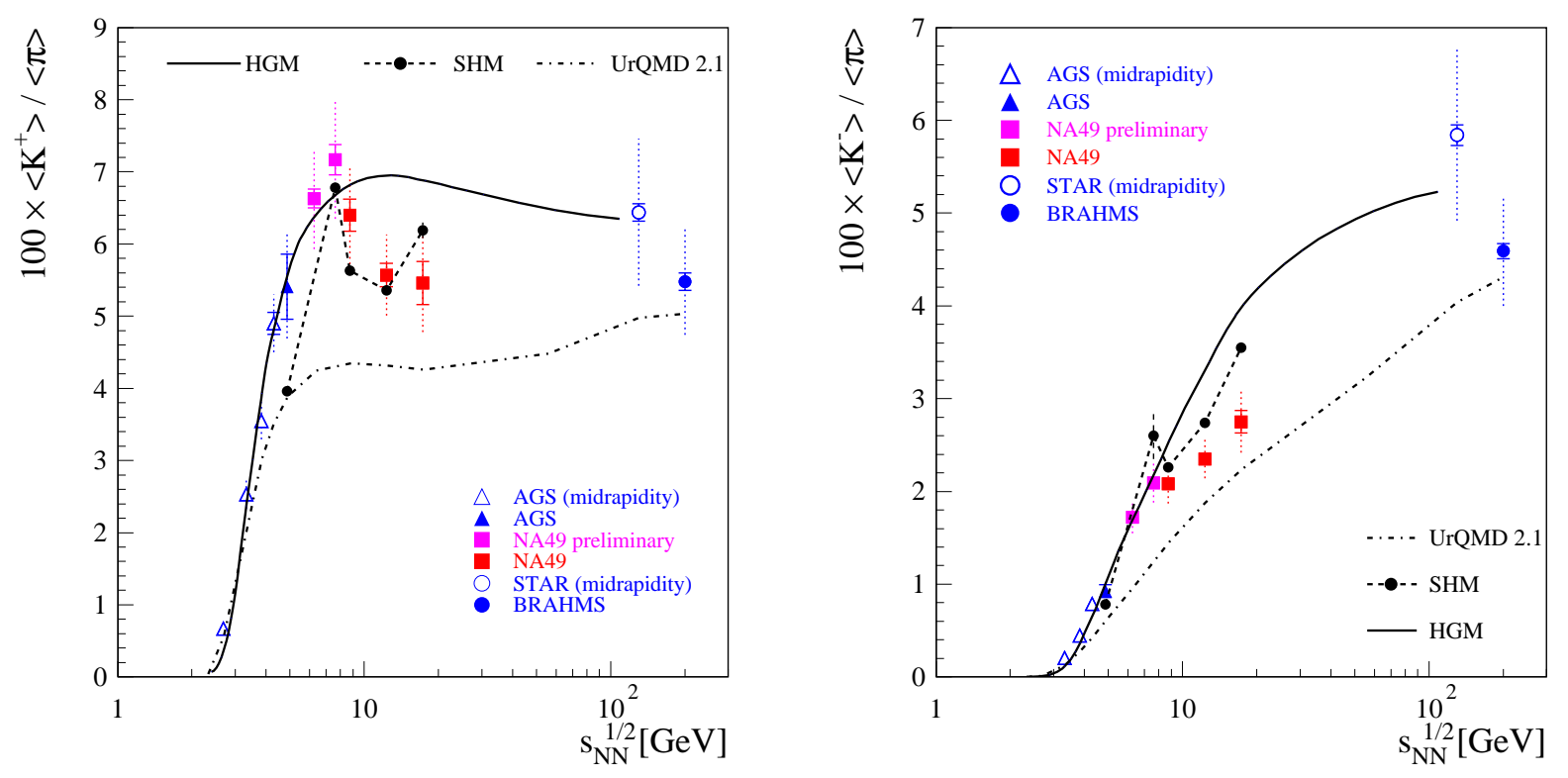

Figure 1. The $K^{+} / \pi$ (left) and $K^{-} / \pi$ (right) ratios in central $\mathrm{Pb}+\mathrm{Pb}(\mathrm{Au}+\mathrm{Au})$ collisions as functions of CM energy. Open symbols denote measurements around midrapidity, closed symbols those in full phase space. The full error bars give the statistical, the dashed ones the sum of statistical and systematic errors. The NA49 data points correspond to 20,30, 40, 80 and $158 \mathrm{AGeV}$ beam energy, respectively. The full line shows the predictions of the HGM, the dots the fit results of the SHM, which are connected by the dashed line to guide the eye. The error of the SHM is derived from the difference between fits A and B in [15]. The dashed-dotted curve represents the results of UrQMD 2.1. Data are NA49 preliminary or taken from [18,20-27]

is no overlap due to the lower momentum cutoff for the $d E / d x$ method. The width of the rapidity distributions increases linearly with the beam rapidity.

By integration of the rapidity distribution, total yields are obtained. The energy dependence of the $\left\langle K^{+}\right\rangle /\langle\pi\rangle$ ratio is shown in figure 1 (left) together with the model results. The newly obtained data point at $20 \mathrm{AGeV}\left(\sqrt{s_{N N}}=6.3 \mathrm{GeV}\right)$ confirms the sharp peak observed in this variable as reported earlier [9]. The HGM, assuming a smooth variation of the $T$ and $\mu_{B}$ parameters with collision energy, is not able to reproduce this feature, although it predicts a broad maximum at low SPS energies. In particular, the relative $K^{+}$yield at higher SPS energies is overpredicted. The SHM, having $\gamma_{S}$ as an additional parameter, is better able to account for the data. UrQMD predicts a small increase in this ratio, but underestimates its value at all SPS energies.

In contrast to the $K^{+}$, the $\left\langle K^{-}\right\rangle /\langle\pi\rangle$ ratio, shown in figure 1 (right), exhibits no sharp structure but a smooth evolution with collision energy, with possibly a small deviation around $30 \mathrm{AGeV}\left(\sqrt{s_{N N}}=7.6 \mathrm{GeV}\right)$. The comparison with the models shows again that HGM overpredicts the data at higher SPS energies. In the SHM fit, the peak in $K^{+} / \pi$ drives $\gamma_{S}$ almost to unity resulting in a similar feature in $K^{-} / \pi$, which is not 

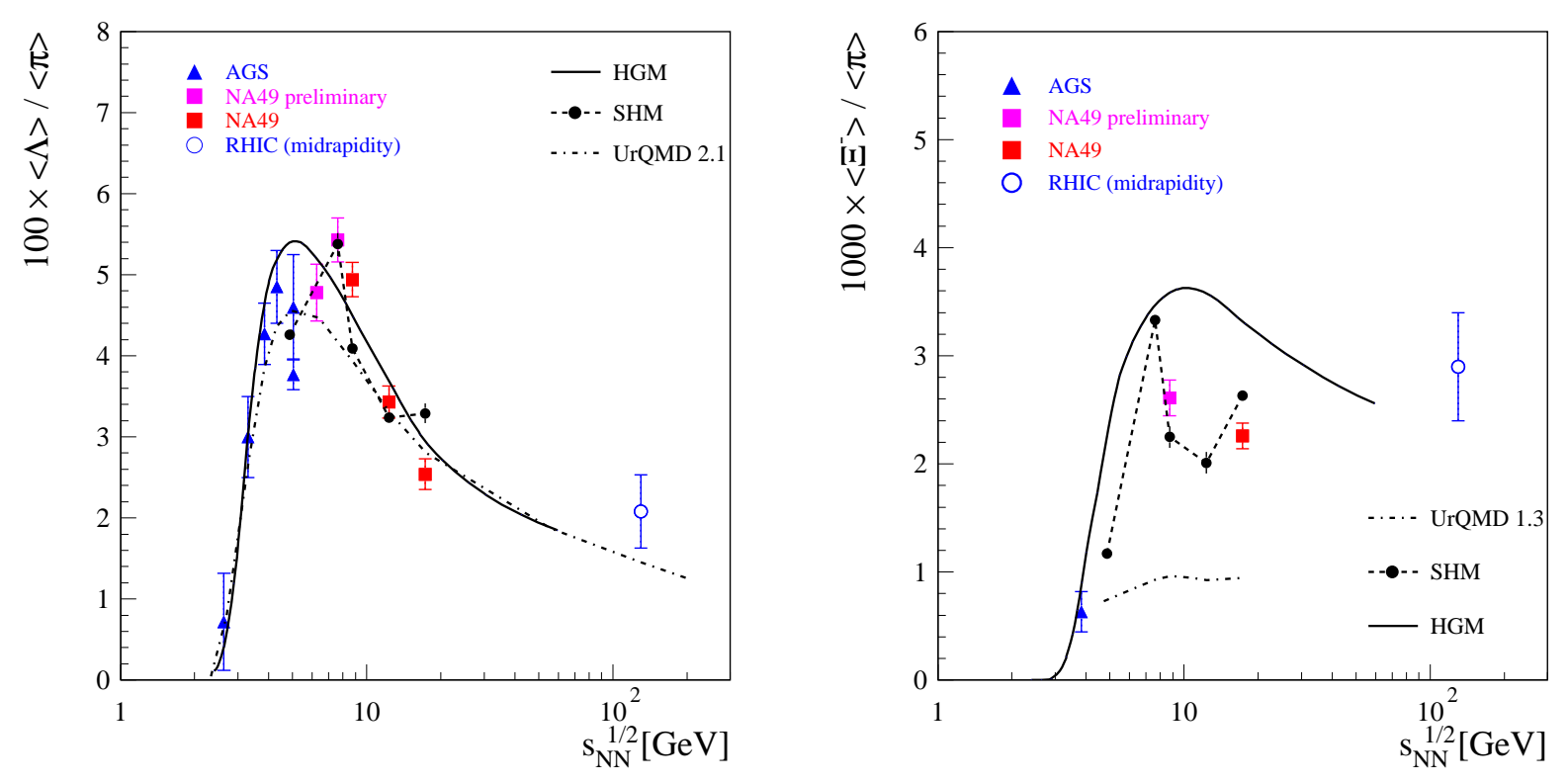

Figure 2. Same as figure $\square$ but for the $\Lambda / \pi$ (left) and $\Xi^{-} / \pi$ (right) ratios. Only statistical errors are shown. Data are NA49 preliminary or taken from $[18,20-23,28-32,34,35]$

as pronounced in the data. The UrQMD results are systematically too low, but closer to the data than in the case of $K^{+} / \pi$.

\section{2. $\Lambda$ and $\bar{\Lambda}$}

The $\Lambda$ and $\bar{\Lambda}$ measurements of NA49 [28] have been extended to the lower SPS energies. While the $\bar{\Lambda}$ rapidity distribution is approximately Gaussian at all energies, the $\Lambda$ distribution visibly flattens above $40 A \mathrm{GeV}[11]$. At top SPS energy, this leads to a larger error in the extrapolation to full phase space.

Normalised to the pion yield, the $\Lambda$ excitation function shows a sharp maximum around $30 \mathrm{AGeV}\left(\sqrt{s_{N N}}=7.6\right)$, similar to the $K^{+}$, although AGS data in this case are not conclusive (figure 2 (left)). As $\Lambda$ and $K^{+}$are the bulk carriers of $s$ and $\bar{s}$ quarks, respectively, and the net strangeness has to vanish, this proves the consistency of the NA49 measurements, the identification methods for kaons and $\Lambda$ being completely different. Although predicting the maximum of $\langle\Lambda\rangle /\langle\pi\rangle$ to be at top AGS energy, the overall agreement of the HGM with the data is reasonable. A still better description is provided by the SHM while UrQMD is close to the measurements at AGS and top SPS but misses the peak at lower SPS energies. 

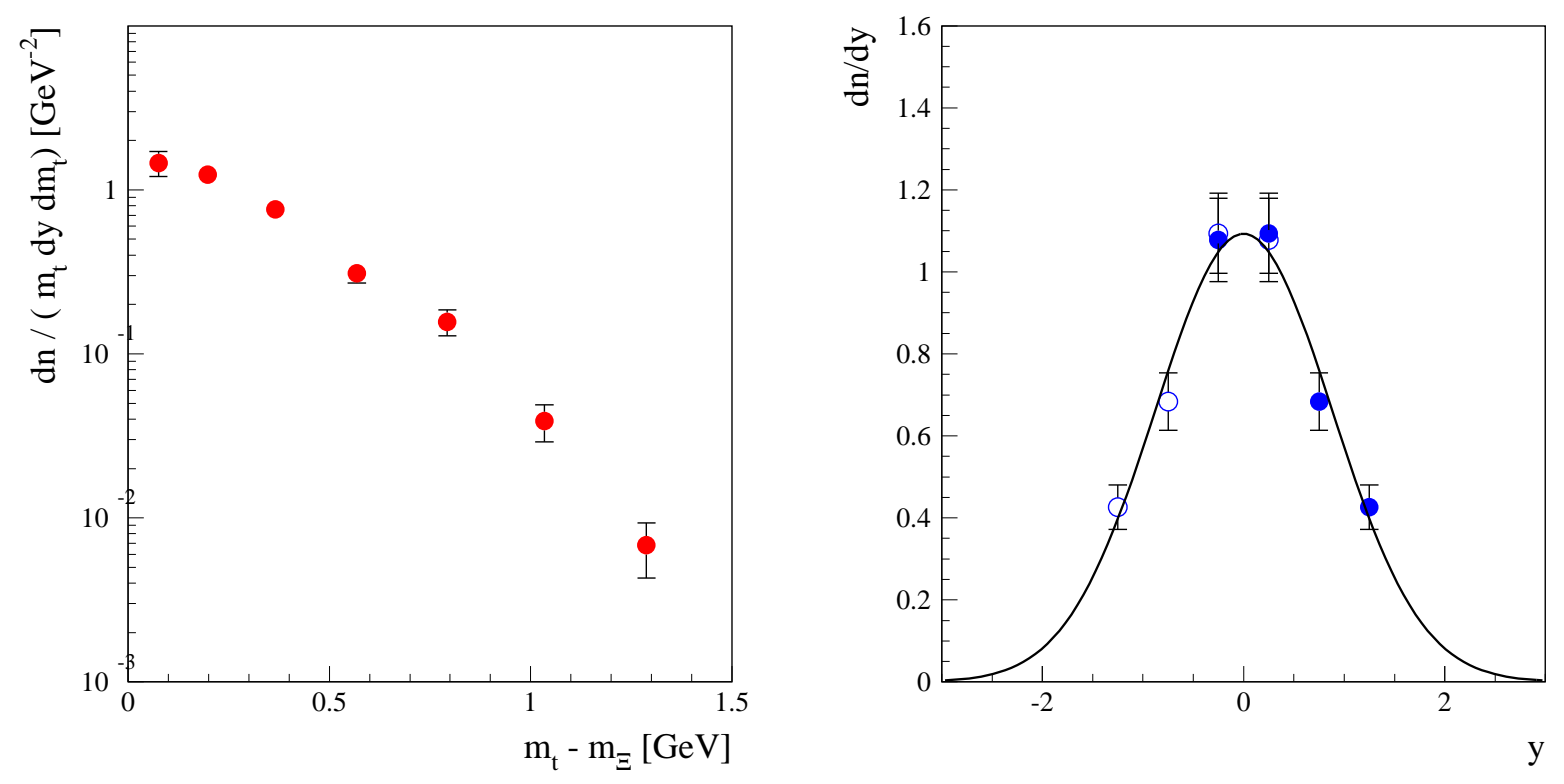

Figure 3. Transverse mass (left) and rapidity (right) spectra of the $\Xi^{-}$in central $\mathrm{Pb}+\mathrm{Pb}$ collisions at $40 \mathrm{AGeV}$. Open points are reflected at midrapidity. The full line shows a Gaussian fit.

\section{3. $\Xi^{-}$}

Multiply strange particles, although carrying only a small fraction of the total strangeness, can be expected to discriminate better between statistical models with and without $\gamma_{S}$ since this factor will enter with a higher power into the predicted yields than for kaons and $\Lambda$. Naturally, data for such rare particles are scarce. In addition to the cascade measurement at top SPS energy [32], the $\Xi^{-}$signal has been analysed by NA49 at $40 A \mathrm{GeV}[33]$. The $\Xi^{-}$transverse mass and rapidity distributions are shown in figure 3. Once more, a single Gaussian gives a satisfactory fit to the rapidity spectrum, indicating the diminishing influence of baryon density with increasing strange quark content. As shown in figure 2 (right), the integrated $\Xi^{-} / \pi$ ratio is slightly higher than at $158 \mathrm{AGeV}$. Both data points are overpredicted by the HGM while again, the SHM is closer to the measurements. UrQMD by far cannot account for the measured yields.

The NA49 cascade analysis is still in progress; promising signals are seen at all beam energies. The excitation function thus can be expected to be better mapped in the near future.

\section{4. $\Omega^{-}$and $\bar{\Omega}^{+}$}

For the $\Omega$ measurement at $158 \mathrm{AGeV}$, the high statistics run of 2000 with a $23.5 \%$ online centrality trigger had to be exploited. At $40 \mathrm{AGeV}$ with considerably less statistics, only the combined $\Omega^{-}+\bar{\Omega}^{+}$signal could be studied. Figure 4 shows the rapidity distributions 

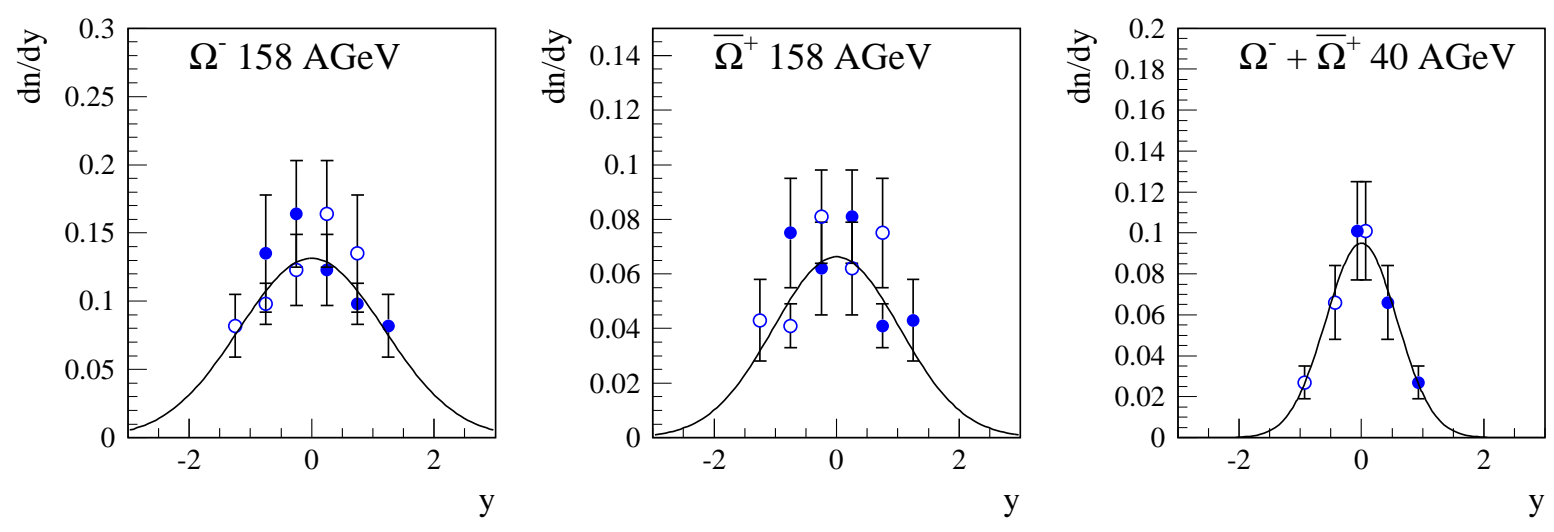

Figure 4. Rapidity distributions for $\Omega^{-}$and $\bar{\Omega}^{+}$at $158 \mathrm{AGeV}$ and for $\Omega^{-}+\bar{\Omega}^{+}$at $40 \mathrm{AGeV}$. Open points are reflected at midrapidity. The full lines show Gaussian fits to the distributions.

for both energies. Within the considerable uncertainties, a Gaussian function gives a good description. As shown in figure 5 (left), the yield ratio $\left(\Omega^{-}+\bar{\Omega}^{+}\right) / \pi$ shows a steady increase from $40 \mathrm{AGeV}\left(\sqrt{s_{N N}}=8.8 \mathrm{GeV}\right)$ over top SPS energy to RHIC. Both flavours of statistical models manage to describe the data within the experimental errors. The small difference between the model results is surprising since naively, one would expect them to differ noticeably, the factor $\gamma_{S}$ entering to the third power. As already observed in the case of cascades, the relative $\Omega$ yield is underestimated strongly by UrQMD.

\section{5. $\phi$ mesons}

In the context of strangeness production, the $\phi$ meson is of special interest because of its strangeness neutrality as a hadron. It should therefore not be sensitive to canonical effects if strangeness production is assumed to happen in a hadronic scenario. It is also not clear whether a possible undersaturation factor $\gamma_{S}$ should be applied or not.

The NA49 $\phi$ measurement at 158 AGeV [37] has been extended to all available energies. Figure 5 (right) shows the normalised $\phi$ yield as function of collision energy. The excitation function is similar to that of the $K^{-}$, showing an overall increase with beam energy. A careful study of the systematic errors is required to assess the significance of the apparent local minimum at $30 \mathrm{AGeV}\left(\sqrt{s_{N N}}=7.6 \mathrm{GeV}\right)$. As in the case of $K^{-}$, the HGM overpredicts the yield at higher SPS energies, while the SHM fits are able to reproduce the data with the exception of $30 \mathrm{AGeV}$. At that energy, the model fit is driven to a maximum as in the case of the $K^{+}$, but the contrary is seen in the data. The $\phi$ yield in UrQMD is not straightforwardly defined since $\phi$ decays happen not only after chemical freezeout but in all stages of the simulated collision, and the rescattering of the decay products will affect the number of detectable $\phi$. 

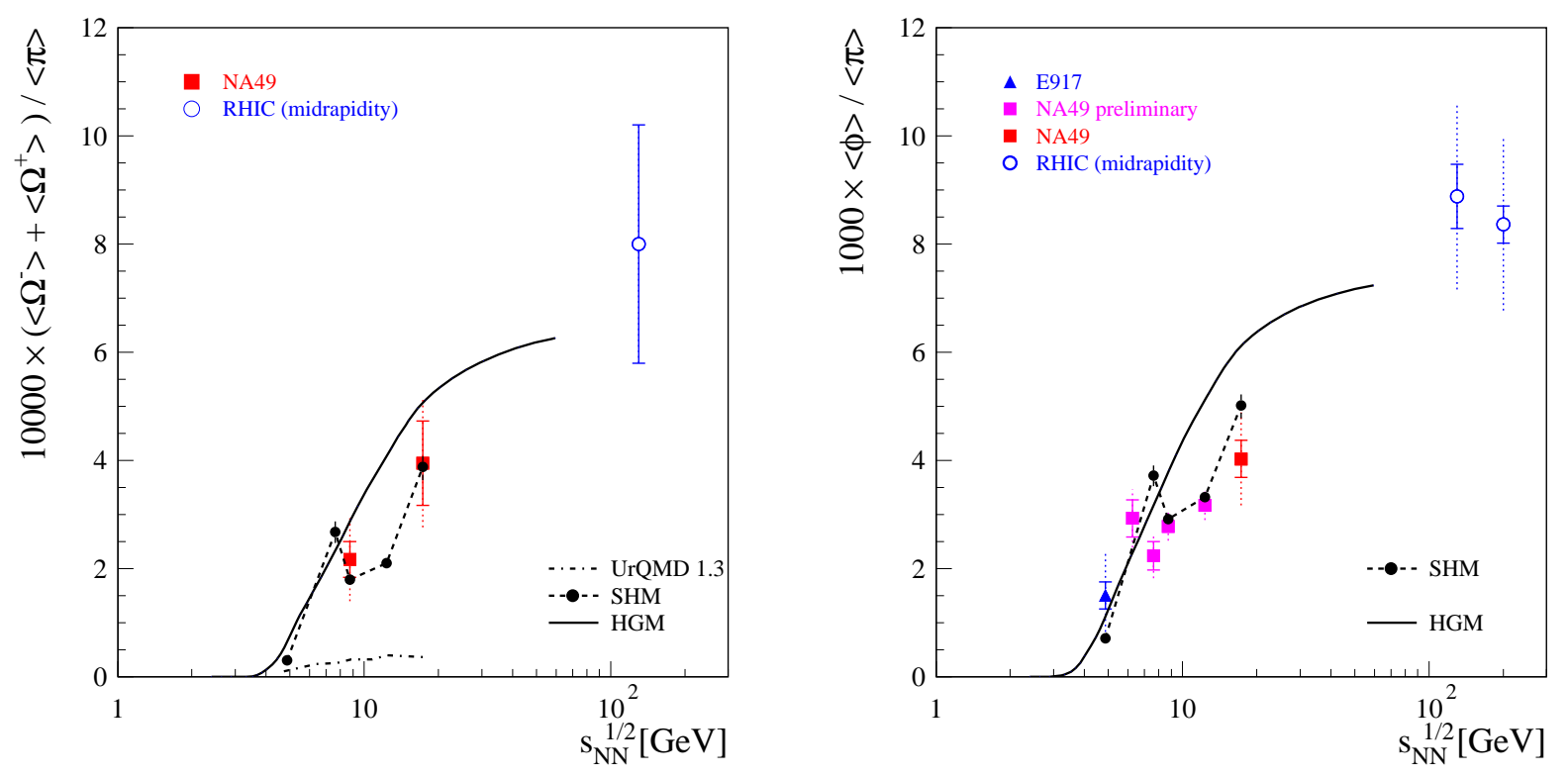

Figure 5. Same as figure $\square$ but for the $\Omega / \pi$ (left) and $\phi / \pi$ (right) ratios. Data are NA49 preliminary or taken from [18,21-23,36-40]

\section{Discussion}

Summarising the comparison of measured particle yields with the models, we find as a general trend that the Hadron Gas Model reproduces the normalised yields at lower collision energies but starts to overpredict them from around $30 \mathrm{AGeV}$ on, with the exception of $\Lambda$ and $\Omega$. At RHIC energies, the measured data are again well reproduced. Apparently, in the SPS energy range the introduction of a strangeness suppression factor $\gamma_{S}$ gives a much better description of the data. At $30 \mathrm{AGeV}$ both model types coincide since here, strangeness seems to be saturated, resulting in $\gamma_{S}$ close to unity. For the other energies, the best fit yields values of $0.7-0.8$ for this parameter. The deviation from the saturation model suggests a basic change in the strangeness production mechanism at around $30 \mathrm{AGeV}$ beam energy. Interestingly, the $\phi$ meson seems to call for $\gamma_{S}$ as well as the other strange hadrons, indicating that $\gamma_{S}$ reflects conditions of a prehadronic stage of the collision. For the interpretation of the $\Omega$ yields, it is unclear why both model types give similar results, contrary to the expectation.

A feature of the HGM is a maximum in the relative strangeness content as a consequence of the interplay between decreasing baryochemical potential and increasing temperature. This maximum, however, is relatively broad and cannot account for the sharp structures seen in the excitation functions of the bulk strangeness carriers. Moreover, its position is shifted towards higher collision energies with increasing strangeness content and is different for mesons and baryons [14]. In this context, the completion of the $\Xi$ excitation function can be expected to have additional 
discriminating power.

In all cases, the UrQMD model underestimates the relative strangeness yields. The discrepancy increases with the strange quark content of the particle; it is largest for the $\Omega$ with three strange valence quarks. For the $K / \pi$ ratio, it can be shown that the discrepancy stems from an overprediction of pions rather than a underestimation of kaons, but this cannot account for the strong underestimation of the multiply strange hadron yields. Apparently, the UrQMD transport model misses some basic features of the underlying strangeness production process.

Considering the total strangeness production in the collision, the sharp peak in the $K^{+} / \pi$ ratio is confirmed by a similar feature in the $\Lambda / \pi$ ratio. It appears doubtful whether such a sharp structure can be explained by a continuous variation of parameters within hadronic models. So far, the only model to predict a sharp maximum in the relative strangeness content is the Statistical Model of the Early Stage [10], assuming the transition to a deconfined state to set in at $30 \mathrm{AGeV}$ beam energy. While this model does not predict the distribution of the produced strangeness among the hadron species, it is obvious that in the high net baryon density environment created at this energy, the $s$ quarks will appear in $\Lambda$ baryons rather than in $K^{-}$, thus explaining the absence of a similar sharp structure in the $K^{-} / \pi$ ratio. We conclude that the data collected by NA49 favour such a scenario.

\section{Acknowledgments}

This work was supported by the US Department of Energy Grant DE-FG0397ER41020/A000, the Bundesministerium für Bildung und Forschung, Germany, the Polish State Committee for Scientific Research (2 P03B 130 23, SPB/CERN/P-03/Dz 446/2002-2004, 2 P03B 04123), the Hungarian Scientific Research Foundation (T032648, T032293, T043514), the Hungarian National Science Foundation, OTKA, (F034707), the Polish-German Foundation, and the Korea Research Foundation Grant (KRF-2003070-C00015).

\section{References}

[1] Stock R 1999 Phys. Lett. B 456277

[2] Heinz U 2001 Nucl. Phys. A 685414

[3] Koch P, Müller B and Rafelski J 1986 Phys. Rep. 142167

[4] Braun-Munzinger P, Heppe I and Stachel J 1999 Phys. Lett. B 46515

[5] Rafelski J and Danos M 1980 Phys. Lett. B 97279

[6] Hamieh S, Redlich K and Tounsi A 2000 Phys. Lett. B 48661

[7] Becattini F et al. 2001 Phys. Rev. C 64024901

[8] Keränen A, Csernai L, Magas V and Manninen J 2003 Phys. Rev. C 67034905

[9] Friese V et el. (NA49 collaboration) 2004 J. Phys. G: Nucl. Part. Phys. 30 S119

[10] Gaździcki M and Gorenstein M 1999 Acta Phys. Pol. B 302705

[11] Blume $\mathrm{C}$ et al. (NA49 collaboration) 2005 these proceedings

[12] Bleicher M et al. 1999 J. Phys. G: Nucl. Part. Phys. 251859

[13] Bratkovskaya E et al. 2004 Phys. Rev. C 69054907 
numerical values supplied by E. Bratkovskaya and M. Bleicher

[14] Braun-Munzinger P et al. 2002 Nucl. Phys. A 697902

numerical values supplied by $\mathrm{K}$. Redlich

[15] Becattini F et al. 2004 Phys. Rev. C 69024905

[16] Röhrich D et al. (BRAHMS collaboration) 2005 these proceedings

[17] Afanasiev S et al. (NA49 collaboration) 1999 Nucl. Instrum. Methods A 430210

[18] Afanasiev S et al. (NA49 collaboration) 2002 Phys. Rev. C 66054902

[19] Gaździcki M et al. (NA49 collaboration) 2004 J. Phys. G: Nucl. Part. Phys. 30 S701

[20] Klay J et al. (E895 collaboration) 2003 Phys. Rev. C 68054905

[21] Ahle L et al. (E802 collaboration) 1998 Phys. Rev. C 57 R466

[22] Adams J et al. (STAR collaboration) 2003 Preprint nucl-ex/0311017

[23] Bearden I et al. (BRAHMS collaboration) 2004 Preprint nucl-ex/0403050

[24] Ahle L et al. (E866 and E917 collaborations) 2000 Phys. Lett. B 4761

[25] Ahle L et al. (E866 and E917 collaborations) 2000 Phys. Lett. B 49053

[26] Ahle L et al. (E802 collaboration) 1998 Phys. Rev. C 583523

[27] Adler C et al. (STAR collaboration) 2004 Phys. Lett. B 595143

[28] Anticic T et al. (NA49 collaboration) 2004 Phys. Rev. Lett. 93022302

[29] Pinkenburg C et al. (E895 collaboration) 2002 Nucl. Phys. A 698 495c

[30] Albergo S et al. (E896 collaboration) 2002 Phys. Rev. Lett. 88062301

[31] Adler C et al. (STAR collaboration) 2002 Phys. Rev. Lett. 89092301

[32] Afanasiev S et al. (NA49 collaboration) 2002 Phys. Lett. B 538275

[33] Meurer C et al. (NA49 collaboration) 2004 J. Phys. G: Nucl. Part. Phys. 30 S1325

[34] Chung P et al. (E895 collaboration) 2003 Phys. Rev. Lett. 91202301

[35] Castillo J et al. (STAR collaboration) 2003 Nucl. Phys. A 715 518c

[36] Suire C et al. (STAR collaboration) 2003 Nucl. Phys. A 715 470c

[37] Afanasiev et al. (NA49 collaboration) 2000 Phys. Lett. B 49159

[38] Back B et al. (E917 collaboration) 2004 Phys. Rev. C 69054901

[39] Adler C et al. (STAR collaboration) 2002 Phys. Rev. C 65 041901(R)

[40] Adams J et al. (STAR collaboration) 2004 Preprint nucl-ex/0406003 